\title{
Téoros
}

Revue de recherche en tourisme

\section{Entretien, avec Jodey CASTRICANO, professeure associée en études critiques (Université de la Colombie-Britannique)}

\section{Alain A. Grenier}

Volume 32, numéro 1, 2013

URI : https://id.erudit.org/iderudit/1036665ar

DOI : https://doi.org/10.7202/1036665ar

Aller au sommaire du numéro

Éditeur(s)

Université du Québec à Montréal

ISSN

0712-8657 (imprimé)

1923-2705 (numérique)

Découvrir la revue

Citer ce document

Grenier, A. A. (2013). Entretien, avec Jodey CASTRICANO, professeure associée en études critiques (Université de la Colombie-Britannique). Téoros, 32(1),

145-147. https://doi.org/10.7202/1036665ar d'utilisation que vous pouvez consulter en ligne. 


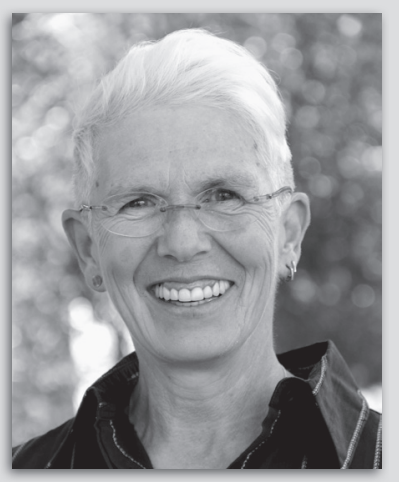

Entretien

avec Jodey CASTRICANO, professeure associée en études critiques (Université de la Colombie-Britannique)

\author{
Propos recueillis par Alain A. Grenier, directeur et rédacteur en chef de Téoros
}

À l'occasion de son dossier spécial sur le tourisme de chasse et pêche, Téoros souhaite offrir à son lectorat le point de vue complémentaire de Jodey CASTRICANO, professeure associée en études critiques à l'Université de la Colombie-Britannique (Faculty of Creative and Critical Studies), et auteure de plusieurs articles et ouvrages sur les questions d'éthique liées aux rapports entre les humains et le monde animal.

\section{À quand remonte votre intérêt pour les questions d'éthique en matière de recherche?}

Même avant d'être une universitaire, je me sentais interpellée par les questions d'environnement et de justice sociale. J'ai commencé à m'intéresser aux aspects éthiques de la recherche sur le bien-être des non-humains pendant ma deuxième année d'université, après avoir vu un documentaire de l'organisme PETA (Pour une éthique dans le traitement des animaux) appelé The Animals Film. Ce documentaire, dont le sous-titre était « $\mathrm{Ce}$ n'est pas eux, c'est nous", dénonçait l'utilisation des animaux dans les secteurs du divertissement, de la recherche scientifique et militaire et de l'agriculture industrielle. II m'a fait prendre conscience de l'ampleur des souffrances infligées aux animaux pour des motifs insignifiants, comme celui de tester des produits ménagers, et à cause desquels des animaux ont subi des brûlures, ont été rendus aveugles ou ont été tués.

C'est à cette époque que j'ai découvert le concept de «spécisme», un terme inventé en 1973 par le psychologue Richard Ryder pour décrire la discrimination généralisée que pratique I'humain envers d'autres espèces. Cette idéologie normalise la dévaluation des non-humains qui est à l'origine de la violence industrielle, scientifique et technologique sans précédent que nous observons de nos jours à l'égard des êtres vivants. J'ai aussi vu, à la même époque, le tristement célèbre documentaire Unnecessary Fuss, produit par Ingrid Newkirsk et Alex Pacheco, de l'organisme PETA. Les images de ce documentaire ont été filmées en 1983 et 1984 à la clinique de traumatismes crâniens de l'université de Pennsylvanie, par les chercheurs eux-mêmes alors qu'ils causaient des lésions cérébrales à des babouins à l'aide d'un dispositif hydraulique. Ce qui m'a vraiment incitée à étudier les problèmes éthiques en recherche est le fait que les chercheurs se moquaient des babouins quand ils leur infligeaient des blessures et, par la suite, devant leur état gravement détérioré. Je me suis alors engagée à parler au nom des êtres non humains qui sont soumis aux pires formes de violence.

\section{Comment intégrez-vous votre approche philosophique à votre travail?}

Quand je suis devenue professeure, j'ai réalisé que je voulais intégrer ces préoccupations éthiques à mes activités de recherche et d'enseignement. En 2003, j'ai mis sur pied un projet, le premier du genre au Canada, qui visait à réunir des experts et des auteurs provenant d'horizons divers et dont les travaux remettent en question les frontières séparant le règne animal de l'humanité, aux niveaux médical, biologique, culturel, philosophique et éthique. L'objet de mon ouvrage, Animal Subjects: An Ethical Reader in a Posthuman World (2008), était de démontrer que, bien que la recherche en matière d'études culturelles avait soutenu la lutte contre le sexisme et le racisme, la question des droits des animaux accusait un retard certain par rapport à l'évolution générale de la société, surtout à l'égard de la souffrance subie par les animaux dans les élevages industriels, les tests de produits, les essais en laboratoire, les zoos, les rodéos, les cirques et les aquariums publics. En ce moment, j'enseigne dans le domaine des études critiques sur les animaux et cette question, comme plusieurs autres, se posent quand on envisage d'étendre les considérations éthiques aux animaux.

\section{Quel serait le principal problème éthique lié aux pratiques de chasse actuelles?}

Chaque année, des millions d'animaux sont tués. L'acte de traiter des êtres vivants comme une ressource ou un trophée constitue une préoccupation éthique grave. II occulte le fait que chaque animal en question est une créature vivante unique, qui possède une valeur intrinsèque. On prétend pratiquer la chasse sportive par amour de la nature. Dans le cas de la chasse de subsistance, elle serait même sensée « offrir au chasseur un lien intime, voire spirituel avec le monde naturel » (Taylor, 2009: 106), au point où la mort de l'animal devient secondaire. En effet, cette activité vise à prendre la vie de l'animal au nom de la communion avec la nature, de l'exercice ou du besoin de tester ses propres compétences en tant que prédateur.

Sans hésitation, et en tant que défenseure de l'ahimsã (non-violence ou respect de la vie), je suis contre la chasse. Je sais que lorsque les chasseurs tuent les animaux, ils le font, disent-ils, pour la nourriture, le sport et le contrôle des populations animales. Mais, à mon avis, il reste que les intérêts vitaux des animaux concernés sont ignorés et que les animaux sont exploités. De nombreux chasseurs affirment que la chasse de subsistance vaut mieux que le soutien de fermes d'élevage, où 
les animaux sont susceptibles de contracter des maladies malgré l'administration d'antibiotiques. La chasse se pratique habituellement dans les pays développés où d'autres sources de nourriture ne manquent pas et où il n'est pas nécessaire de prendre la vie d'un animal.

Par ailleurs, l'utilisation d'armes lors des activités de chasse ne garantit pas que les animaux seront effectivement tués. Des études ont révélé que de nombreux chasseurs moins habiles laissent les animaux blessés souffrir et mourir. La notion d'abattage des animaux est tout aussi problématique, car les animaux doivent rivaliser avec les humains qui empiètent de plus en plus sur leurs habitats et en viennent à les considérer comme des parasites ou des bêtes nuisibles. Quant à la chasse au trophée, j'estime que le meurtre d'un magnifique être vivant, simplement parce que l'on veut stimuler son ego en exposant la tête, les pattes ou le panache d'un animal, est un geste abominable. La vie de ces animaux avait une valeur. Je dis cela parce que de plus en plus de recherches en éthologie cognitive montrent que les animaux sont capables de ressentir toute une gamme d'émotions que l'on croyait précédemment être le propre des humains.

\section{Les avis sont tranchés sur le sujet de la chasse. C'est encore davantage le cas lorsqu'elle est organisée comme une activité lucrative, notamment en tourisme. Quelles questions éthiques peuvent être soulevées par la commercialisation de la chasse récréative par rapport à la chasse de subsistance?}

Étant donné que je ne pense pas que la chasse constitue en soi une pratique éthique, je ne dirai certainement pas que la chasse récréative est une pratique éthique, puisqu'elle viole le principe de la valeur intrinsèque de la vie d'un animal.

Plusieurs chasseurs, y compris des femmes, affirment qu'ils chassent pour des raisons écologiques ou spirituelles. Mais selon Cartmill (1996: 30), par définition, la chasse n'est rien d'autre que «le meurtre délibéré et violent d'animaux sauvages et libres». Le but de l'activité est de rivaliser avec ledit animal. Toutefois, en réalité, l'animal n'a pas donné son consentement à une telle compétition. Ici, la seule finalité de l'exercice est de tuer.

La chasse organisée en tant qu' activité lucrative est problématique sur le plan éthique, surtout lorsque la mise à mort d'un animal est garantie par le voyagiste, comme c'est le cas pour la chasse au couguar, la chasse à l'élan en secteur clôturé, la chasses au trophée à l'ours noir, la chasse à l'ours avec appât ou encore la chasse au sanglier, où chasseurs sont encouragés à considérer l'atteinte de leur cible comme un acte prestigieux. Des organismes comme PETA ont dénoncé le fait que nombre d'animaux qui sont chassés par des personnes sont blessés et ainsi condamnés à une mort due à la faim, à la déshydratation ou à une infection. Au nom du prestige, on tue aussi fréquemment des animaux plus jeunes et plus forts, qui laissent un vide dans la communauté animale. De même, beaucoup d'animaux tués ont des petits, qui sont eux aussi abandonnés à une mort lente.

La chasse peut-elle contribuer au bien-être de la nature? L'idée selon laquelle la chasse et la pêche représentent des moyens de contrôler la croissance des populations animales semble égoïste, surtout quand on sait que des habitats sont détruits et que des espèces sont menacées. On prétend que le fait de tuer des animaux constitue une pratique durable, car il serait nécessaire de réduire la taille des populations sauvages. Cependant, le déséquilibre résulte en fait de la mise à mort des prédateurs, que l'on considère comme des animaux nuisibles.

Depuis longtemps, on perçoit la chasse et la pêche comme des pratiques masculines. L'augmentation du nombre de femmes participant à ces activités modifiera-t-elle la pratique de la chasse?

II est vrai que le nombre de femmes participant à des activités de chasse a augmenté, mais rien ne m'indique comment cela changerait la façon dont la chasse est pratiquée. Diverses études ont établi une corrélation entre la virilité et l'exploitation des animaux. Plusieurs femmes, dont certaines se décrivent comme d'anciennes végétariennes, prennent les armes pour avoir une viande qui est tuée le plus éthiquement possible. Je trouve qu'il y a quelque chose de malhonnête là-dedans, car cela semble suggérer que l'éthique a une limite quand il s'agit de prendre une vie. La question devient alors : que signifie tuer «le plus éthiquement possible» face à l'intégrité morale?

\section{Puisque la chasse et la pêche requièrent une bonne connaissance du comportement animalier, plusieurs personnes estiment que ces activités sont aussi valables que l'observation et permettent une reconnexion avec la nature.}

À mon avis, une telle affirmation n'est qu'une stratégie visant à légitimer le désir de chasser. Si l'observation était vraiment le but de l'exercice, les gens pourraient se munir d'une caméra et aller « reconnecter» avec la nature sans perpétrer aucune violence.

Les militants contre la chasse font souvent une distinction entre les espèces. Pourquoi?

Bien que la distinction entre les espèces puisse être une forme de spécisme, elle est faite simplement parce que l'on est enjoint, souvent au niveau local, de s'opposer à la chasse lorsqu'une espèce est particulièrement à risque. Par exemple, certains militants s'inquiètent de la mise à mort des loups, qu'ils considèrent comme un massacre rémunéré. Je ne pense pas que la distinction entre les espèces signifie que les militants ne se soucient pas des autres espèces. Ce serait comme dire qu'une personne qui se soucie des animaux ne se soucie pas des humains. En fait, la plupart des personnes impliquées dans la protection des animaux au $19^{e}$ siècle étaient celles-là mêmes qui ont participé à la création de lois contre la cruauté envers les enfants.

Certaines espèces emblématiques, comme l'ours polaires et les baleines, attirent beaucoup l'attention des médias et des défenseurs des animaux. Qu'en est-il des espèces menacées qui pourraient ne pas être aussi photogéniques?

Si les médias se concentrent sur les ours polaires et les baleines, c'est en raison d'une vision spéciste des autres formes de vie sur cette planète, qui sont considérés comme inférieures. Les médias incitent également les gens à croire que certains animaux méritent davantage notre protection que d'autres. Beaucoup de gens estiment qu'il est plus important de protéger des espèces emblématiques, comme les baleines, les tigres et les ours polaires, que, par exemple, les serpents, les grenouilles ou d'autres animaux couverts d'écailles. Pourquoi? Ce pourrait 
être parce que ces derniers sont moins photogéniques, et que d'autres espèces nous sont présentées comme étant nobles et dignes de notre protection. Ce raisonnement se situe dans le même registre que la dissonance cognitive qui permet aux gens d'avoir un chien ou un chat comme animal compagnie, mais d'en abattre d'autres, surtout quand ceux-ci ne sont pas considérés comme des individus.

\section{Le discours sur la protection et le respect de la faune est souvent orienté vers la protection des mammifères. Les poissons semblent avoir beaucoup moins attirer la sympathie du public. Devrait-on aussi se préoccuper des poissons?}

II est vrai que les gens ne croient pas que les poissons puissent ressentir de la douleur ou du plaisir. Toutefois, des études ont prouvé que cela est faux et que, en fait, non seulement les poissons ressentent la douleur et le plaisir, mais qu'ils ont aussi des vies émotionnelles complexes. Les gens ne partagent pas cette vision, peut-être parce qu'on ne considère pas que les poissons ont des expressions faciales. Cependant, les scientifiques ont démontré que les poissons rouges, par exemple, ont la capacité d'apprendre. II y a quelques années, nous appliquions la même forme de déni face aux poulets, mais la science a depuis révélé que ces oiseaux peuvent obtenir de meilleurs résultats que les chiens ou les chats lors de tests cognitifs. En d'autres mots, les poulets ont montré des niveaux de conscience de soi supérieurs à ceux qu'on leur attribuait traditionnellement. Pourtant, ils demeurent, comme les dindes, au nombre des animaux les plus mal traités par le secteur de l'élevage industriel. La façon dont les animaux sont traités dépend de notre perception à leur égard. Notre vision des dauphins et des baleines a évolué et pourrait bien, un jour, s'étendre aux poissons.

\section{Si la chasse est contraire à l'éthique, et si on ne peut pas devenir végétarien, quelles solutions avons-nous en tant que consommateurs?}

II existe un mythe selon lequel le mode de vie végétarien ne fournit pas suffisamment de protéines, mais environ 20 millions d'Américains sont végétariens ou végétaliens. Moins de $1 \%$ des animaux tués pour leur viande proviennent de petites fermes familiales. Les fermes industrielles modernes maintiennent généralement les vaches, les veaux, les cochons, les poulets, les dindes, les canards et autres dans des espaces surpeuplés, où ils ne peuvent même pas se retourner. Ces animaux sont, bien entendu, régulièrement soumis à de mauvais traitements et à des injections d'antibiotiques.

Tant d'animaux meurent afin que les gens puissent manger de la viande sous forme de hamburgers, de rôtis, de hot dogs, de bacon, mais tous ces termes cachent la réalité de l'abattage. Les protéines animales (y compris les produits laitiers) sont en fait liées aux maladies chroniques, aux maladies coronariennes et à certaines formes de cancer. Par exemple, deux études de l'université Harvard ont démontré que les hommes qui boivent du lait couraient $60 \%$ plus de risques de développer un cancer de la prostate que ceux qui évitent les produits laitiers. II est également important de reconnaitre que la consommation de protéines animales n'a jamais été une nécessité biologique, bien que l'on dise le contraire. En réalité, on mange de la viande parce que l'on nous a appris à le faire.
Quelles solutions avons-nous en tant que consommateurs? L'élevage d'animaux à des fins alimentaires se termine sur le plancher des abattoirs. Soyez conscient des activités que vous soutenez au nom du plaisir, alors que d'autres sources de protéines de meilleure qualité et plus respectueuses de l'environnement sont à votre disposition.

\section{Références}

CARTMILL, Matt (1996) A View to a Death in the Morning - Hunting and Nature Through History, Harvard University Press, 347 pages.

TAYLOR, Angus (2009) Animals and Ethics: An Overview of the Philosophical Debate, $3^{e}$ edition, Broadview Press, Peterborough, Ontario. 232 pages.

\section{POUR EN LIRE PLUS (suite de la page 144)}

Le livre, tantôt très universitaire, tantôt plus politique, s'adresse à un lectorat ouvert et prêt à revoir le modèle actuel des rapports quasi institutionnels avec les animaux. On y aborde autant des questions philosophiques, comme celles de la situation des animaux ("A Missed Opportunity: Humanism, Anti-Humanism and the Animal Question», Paola Cavalieri), de la nécessité de penser autrement («Thinking Other-Wise: Cognitive Science, Deconstruction and the (Non)Speaking (Non)Human Animal Subject", Cary Wolfe), du blâme et de la honte ("Blame and Shame? How Can We Reduce Unproductive Animal Experimentation?», Anne Innis Dagg), que des sujets plus pratiques, comme l'utilisation des animaux dans les domaines récréatif et touristique («Monsters: The Case of Marineland», John Sorenson).

À partir d'exemples et d'une recension élaborée d'ouvrages contemporains et plus anciens, Castricano et ses collègues retracent ici les origines des conventions qui régissent nos rapports avec les animaux. Ils démontrent comment, en les dénigrant à des rangs inférieurs, nous arrivons aujourd'hui à ne pas nous soucier de leur bien-être et à en abuser dans le cadre de nos activités quotidiennes, ou plus indirectement par nos choix de consommation, qui engendrent une exploitation à outrance du monde animal au nom de la supériorité humaine.

Alain A. Grenier, Ph.D., Département d'études urbaines et touristiques (ESG-UQAM) 